Draft version August 26, 2018

Preprint typeset using $\mathrm{LAT}_{\mathrm{E}} \mathrm{X}$ style emulateapj v. $7 / 15 / 03$

\title{
DYNAMICAL CONSTRAINTS ON THE ORIGIN OF THE YOUNG B-STARS IN THE GALACTIC CENTER
}

\author{
Hagai B. Perets ${ }^{1}$ And Alessia Gualandris ${ }^{2}$ \\ Draft version August 26, 2018
}

\begin{abstract}
Regular star formation is thought to be inhibited close to the massive black hole $(\mathrm{MBH})$ in the Galactic center. Nevertheless, tens of young main sequence B stars have been observed in an isotropic distribution close to it. These stars are observed to have an apparently continuous distribution from very close to the $\mathrm{MBH}<0.01 \mathrm{pc}$ and up to at least $\sim 0.5 \mathrm{pc}$, suggesting a common origin. Various models have been suggested for the formation of the B-stars closest to the MBH $(<0.05 \mathrm{pc}$; the Sstars), typically involving the migration of these stars from their original birthplace to their currently observed position. Here we explore the orbital phase space distribution of the B-stars throughout the central $p c$ expected from the various suggested models for the origin of the B-stars. We find that most of these models have difficulties in explaining, by themselves, both the population of the S-stars $(<0.05 \mathrm{pc})$, and the population of the young B-stars further away (up to $0.5 \mathrm{pc})$. Most models grossly over-predict the number of B-stars up to $0.5 \mathrm{pc}$, given the observed number of S-stars. Such models include the intermediate-mass black hole assisted cluster inspiral scenario, Kozai-like perturbations by two disks, spiral density waves migration in a gaseous disk, and some of the eccentric disk instability models. We focus on one of the other models, the massive perturber induced binary disruption, which is consistent with both the S-stars and the extended population of B-stars further away. For this model we use analytical arguments and N-body simulations to provide further observational predictions. These could be compared with future observations to further support this model, constrain it or refute it. These predictions include the radial distribution of the young B-stars, their eccentricity distribution and its dependence on distance from the $\mathrm{MBH}$ (higher eccentricities at larger distances from the MBH), as well as less specific expectations regarding their mass function.
\end{abstract}

Subject headings:

\section{INTRODUCTION}

In recent years high resolution observations have revealed the existence of many young $\mathrm{O}$ and $\mathrm{B}$ stars in the galactic center (GC). Accurate measurements of the orbital parameters of these stars give strong evidence for the existence of a massive black hole $(\mathrm{MBH})$ which governs the dynamics in the GC (Ghez et al. 1998; Eisenhauer et al. 2005; Gillessen et al. 2009). Most of the young stars are observed in the central $0.5 \mathrm{pc}$ around the $\mathrm{MBH}$. The young stars population in the central $\sim \operatorname{arcsecond}(0.05 \mathrm{pc})$, the so called 'S-stars', contain only young B-stars (likely masses of $7-15 M_{\odot}$ ), in apparently isotropic distribution around the $\mathrm{MBH}$, with relatively high eccentricities (consistent with a thermal distribution, with $0.3 \leq e \leq 0.95$; Gillessen et al. 2009). The young stars outside this region contain many O-stars and present markedly different orbital properties, with a top heavy mass function and a disk (or disks) like configuration(s) (Levin \& Beloborodov 2003; Lu et al. 2009; Bartko et al. 2009, 2010), and much more circular orbits. The sharp difference and discontinuity between the O-stars population outside the central $0.05 \mathrm{pc}$, and the S-stars population inside this region strongly suggest a different origin for these two populations (see also Bartko et al. 2010).

Recently, it was found that the isotropically distributed

Electronic address: hperets@cfa.harvard.edu

${ }^{1}$ CfA fellow, Harvard-Smithsonian Center for Astrophysics, 60 Garden St., Camridge, MA 02138, USA

${ }^{2}$ Max-Planck Institut für Astrophysik, Karl-Schwarzschild-Str. 1, D-85741 Garching, Germany
B-stars population extends (apparently) continuously beyond the central arcsecond and up to at least $0.5 \mathrm{pc}$ from the $\mathrm{MBH}$ (Bartko et al. 2010). Although many studies have explored the origin of the 'S-stars' (see Perets et al. 2009), the observations of the extended population may shed new light on the origin of these stars. Given the seemingly continuous nature of the B-star population, it is likely that this population of both the S-stars and the extended population at larger distances (or at least most of it), has a single origin. In the following we make this assumption and study possible scenarios for a single common origin of all the GC B-stars and their later evolution. We discuss and suggest specific observational constraints on the various models. We then mainly focus on one of these models, the massive perturbers induced binary disruption (Perets et al. 2007), and use analytical arguments and N-body simulations to provide specific predictions and observational signatures expected from this model.

\section{ORIGINS AND EVOLUTION}

Regular star formation in the close region near the $\mathrm{MBH}$ is inhibited by the tidal forces which destroy any potential giant molecular cloud progenitor. For this reason, young stars in this region are thought to either form elsewhere, further away from the $\mathrm{MBH}$, and then migrate rapidly closer to the $\mathrm{MBH}$; or form in-situ near the $\mathrm{MBH}$, in an irregular form of star formation, most likely through instabilities in a gaseous disk (see below). After their formation/capture, stars in the GC environment can dynamically evolve. Each of the scenarios for the B-stars origin produces a different and unique distri- 
bution of their initial orbital configurations. These configurations, however, can later dynamically evolve due to relaxation processes. The currently observed properties of these stars therefore reflect the combination of the initial configurations and their later evolution. In the following we discuss the various models for the origin of the B-stars. We briefly review each of them, discuss their possible caveats, and detail dynamical constraints which could be used to exclude or support these models. As we shall see, simple efficiency arguments show that most of the suggested models for the B-stars origin can already be strongly constrained, if not excluded, by current observations.

Note that although we focus on the origin of the B-stars in the GC, in the following we also mention scenarios for the disk $\mathrm{O}$ and WR-stars origin, since their population is closely related to that of the B-stars in some of the models we discuss.

\subsection{In-situ star-formation from infalling gaseous clumps}

It was suggested that the young OB stars in the GC were formed in-situ in the central pc a few million years ago in gaseous disks and/or streams formed from infalling and/or colliding gaseous clumps (Morris 1993). Analytic calculations and simulations (Nayakshin \& Cuadra 2005; Levin 2007; Bonnell \& Rice 2008; Wardle \& Yusef-Zadeh 2008; Hobbs \& Nayakshin 2009) have shown that stars could form in such fragmenting clumps to produce stellar disks and/or other coherent structures, in the region of a few 0.01 pcs up to a few 0.1 pcs from the MBH. These could, in principle, be consistent with the observed young stellar structures (clockwise disk and a secondary inclined structure). This scenario could also explain the radial distribution of stars in the observed stellar disk and possibly their eccentricities. Moreover, some of the proposed models produce a top heavy mass function (MF) for the newly formed stars, possibly consistent with observations (Bartko et al. 2010). None of these, however, produces the population of B-stars within $0.05 \mathrm{pc}$ of the $\mathrm{MBH}$

One should note that our poor understanding of the initial conditions and the star formation processes in the $\mathrm{GC}$, allows for a wide parameter space to choose from, which, naturally, raises difficulties in constraining (or strictly falsifying) such models. Nevertheless, the robustness of producing some sort of star formation in the GC region under a variety of conditions explored in the literature suggest these models as the currently most promising scenarios for the origin of the young stellar disk and stellar structures (although less likely the origin of the isotropic B-stars population, including the S-stars, as discussed below).

\subsection{In-situ star-formation followed by rapid migration}

None of the models for in-situ star formation in the central pc suggests the formation of stars as close to the $\mathrm{MBH}$ as the S-stars, or the apparent existence of two distinct young $\mathrm{O}$ and $\mathrm{B}$ stellar populations. Producing both the disk and isotropic B-stars population in the same scenario (and in particular the inner S-stars) requires some fine-tuning. Specifically, comparing the top heavy mass function of the disk stars, as suggested by current observations, with the more regular mass function of the isotropic B-stars disfavors a common origin. One therefore requires a selective process which works differentially on stars of different masses. In any case, an additional process would be required for the migration of stars from the outer to inner regions in the central pc in order to produce the S-stars close to the $\mathrm{MBH}$.

Two-body relaxation processes work, in principle, differentially on stars of different masses, through mass segregation (energy equipartition). For example, relaxation in an isolated stellar disk would somewhat segregate the more massive stars into a thinner disk (Alexander et al. 2007; Perets et al. 2008b; Löckmann et al. 2009), producing mass stratified populations. However, this effect is relatively small. Moreover, the most important component for relaxation in the GC is likely to be the stellar black holes population in the stellar cusp (Perets et al. 2008b; Löckmann et al. 2009; Perets et al. 2009), which is dominated by resonant relaxation (Perets et al. 2009). Segregation into two different stellar populations of different mass regimes is not likely to ensue in this case. Moreover, such scattering of stars could not produce the population of S-stars closer to the MBH (Perets et al. 2008b; also Perets et al., in prep.). Encounters of binarysingle stars (Cuadra et al. 2008; Perets et al. 2008a) can also only have a small effect in producing the isotropic Bstars population from a thin stellar disk. Perturbations by massive perturbers (Perets et al. 2007) such as infalling intermediate-mass black holes (IMBHs) (Yu et al. 2007; Gualandris \& Merritt 2009) or other stellar disks (Löckmann et al.|2009, ; also Gualandris et al., in prep.) do not differentiate between stars of different masses either.

\subsubsection{Efficiency constraints on in-situ formation of the $B$-stars in the central $p c$}

Given the lacking suggestion for a mass differentiating process, a different route can be taken to explain the different stellar populations. We can suggest that two distinct epochs of in-situ star-formation occurred. Two such epochs would still require migration of some of the stars from the disk to the inner region, where stars do not form in-situ. One should mention, in this context, that in some cases a different mass function was found for stars formed in different structures in the same simulation (Hobbs \& Nayakshin 2009). We can therefore either suggest two epochs of star formation happening at different times, or a single epoch producing two distinct populations. In either case, the rapid migration producing the S-stars should affect only one of the stellar populations formed.

Two distinct star formation epochs could naturally produce more massive and less massive stellar populations. Even if both epochs produced stellar populations with the same initial mass function, the most massive stars from the first epoch might already have ended their life, leaving behind a stellar population of less massive stars. This scenario could be constrained by observations. We may suggest an interesting and very general requirement applicable to any scenario in which the S-stars formed far from their current positions. In any such scenario only a fraction $f_{m i g}$ of the stars formed in some external region (e.g. the stellar disk, the central pc, or regions outside the central pc) finally migrate to become S-stars. For a given number of observed S-stars, $N_{s}$, the parent 
external population should be $1 / f_{\text {mig }}$ larger to have

$$
N_{\text {par }}=N_{s} / f_{\text {mig }}
$$

stars (compare with similar constraints derived by Perets 2009a). Cases in which $f_{m i g}$ is small could be strongly constrained by such requirement. For example, the 'billiard' model for the origin of the S-stars (Alexander \& Livio 2004) in which stars from the central pc are captured close to the MBH through exchanges with SBHs close to the $\mathrm{MBH}$, is disfavored since the population of similar B-stars in the central $\mathrm{pc}$ is too small to accommodate the required parent population (Paumard et al. 2006). Similarly, one can turn to models of a disk origin for the S-stars, such as the Kozai-like perturbations in the two disks scenario (Löckmann et al. 2009), the eccentric disk instability model (Madigan et al. 2009), or the spiral density wave model (Griv 2010). All of these models, irrespective of details, suggest that the S-stars formed as part of a stellar disk, up to a few $0.1 \mathrm{pc}$ away from the $\mathrm{MBH}$. A small fraction of the stars formed in the disk migrated through some process to later on become the currently observed S-stars. The current number of B-stars inferred in the central $0.5 \mathrm{pc}$, but outside the central $0.05 \mathrm{pc}$ where the S-stars reside, is comparable, and likely somewhat smaller than the number of S-stars. These models are therefore required to have a very high migration efficiency in order to explain the origin of the S-stars. In table 1 we list the expected number of B-stars in the central $0.05 \mathrm{pc}$ and those up to $\sim 0.5 \mathrm{pc}$, according to the various models. We compare these numbers with the observationally inferred numbers. We also list some of the non in-situ formation models, which are discussed later on. Most of the current formulations of the models are likely excluded, as can be seen from the table. Whether similar models could still be consistent with observations under somewhat different conditions than currently studied, would require additional investigation.

\subsection{External star-formation followed by rapid migration}

Another set of scenarios suggest that the GC young stars formed outside the central pc, where conditions are less hostile to regular star formation. These scenarios could be used to explain either the origin of the stellar disk, the origin of the isotropic B-stars, in particular the S-stars, or both. Note that the extension of S-stars distribution beyond the central $0.05 \mathrm{pc}$ is only a recent observational development, nevertheless, some of the suggested models discuss the possible existence of such an extended distribution.

\subsubsection{Cluster and IMBH infall}

An infall of a young stellar cluster (with or without an IMBH) into the central pc was suggested as an alternative scenario for the origin of the $\mathrm{GC}$ young stars (Gerhard 2001; Hansen \& Milosavliević 2003; Kim \& Morris 2003; Portegies Zwart et al. 2003; Kim et al.|2004; Levin et al. 2005; Gürkan \& Rasio 2005; Berukoff \& Hansen 2006; Fujii et al. 2008, 2009). Such dissolving cluster is likely to form a stellar disk-like structure, possibly with additional outlying structures and/or isolated stars outside the main disk, as observed in the GC. It would also produce a bias towards more massive stars being concentrated in the central region of the GC. These massive stars, which were originally segregated in the innermost regions of the cluster, would be the last to evaporate from the cluster, i.e. in the central most regions closer to the $\mathrm{MBH}$. In addition, an IMBH infall has the potential to deplete the inner regions of the $\mathrm{GC}$, and form a core-like structure (Löckmann \& Baumgardt 2008; Merritt 2009), as possibly observed (Buchholz et al. 2009; Do et al. 2009; Bartko et al. 2010). Yu et al. (2007) also invoke the existence of an IMBH with mass $>10^{4} M_{\odot}$ to help produce higher eccentricities and inclinations for the disk stars. An infall of an IMBH could also rapidly isotropize the distribution of stars closest to the $\mathrm{MBH}$, possibly helping explain the currently observed distribution of the S-stars in the central 0.05 pc (Merritt et al. 2009; Gualandris \& Merritt 2009). These properties make this scenario quite attractive. Nevertheless, the inspiral scenario also has many difficulties, especially in regard to the origin of the B-stars on which we focus here. We shortly discuss these in the following.

Infalling clusters may not be able to inspiral in the appropriate time window for producing the stellar disk. Kim \& Morris (2003) show that a cluster (without an $\mathrm{IMBH}$ ) would need to be extremely dense or born within the central $5 \mathrm{pc}$ to inspiral fast enough. Kim et al. (2004) find that the presence of an IMBH lessens the requirements on the cluster density, if it is as massive as $10 \%$ of the cluster mass, however Gürkan et al. (2004) find that typical masses for IMBHs formed in simulations of dense clusters (without stellar evolution) are of the order of $10^{-3}$ of the cluster mass. The formation of appropriate IMBHs in star clusters may therefore be very difficult. Moreover, even the formation of lower mass IMBHs through collisions in dense clusters is found to be difficult when stellar evolution and wind mass loss are taken into account (Yungelson et al. 2008; Glebbeek et al. 2009; Vanbeveren et al. 2009). The IMBH cluster infall model is therefore unfavorable given our current understanding. The mass and orbital separation of a possible IMBH in the GC are constrained by radio observations of SgrA* (see Gualandris \& Merritt 2009, for a recent overview of these constraints).

Another potential issue with the infall model is that an inspiraling cluster is likely to leave most of its stars behind as it dissolves, whereas very few young stars are observed outside the central 0.5 pc. Simulations of an infalling cluster hosting an IMBH (Levin et al. 2005; Berukoff \& Hansen 2006; Fujii et al. 2010) show that the young stars are stripped from the cluster before the IMBH reaches the central $0.05 \mathrm{pc}$. The young stars closest to the MBH (the S-stars) are therefore not likely to directly originate from such a scenario. Starting from a cluster of 50 stars strongly bound to an inspiraling IMBH (separation of up to $0.06 \mathrm{pc}$ ), Berukoff \& Hansen (2006) showed that at most 1-2 stars could reach distances of $0.04 \mathrm{pc}$ from the $\mathrm{MBH}$. In other words, $96-98$ percent of the stars were stripped off when the IMBH was at distances of a few $0.1 \mathrm{pc}$ from the central $\mathrm{MBH}$. In terms of the "migration efficiency" defined above, we get $f_{m i g} \leq 0.04$, where we have replaced the parent population of the newly formed stars in the disk with the parent population of the stars originally closely bound to the IMBH. Similar results are obtained by Fuiji et al. 


\begin{tabular}{|c|c|c|c|c|c|}
\hline Model & $f_{m i g}$ & $\begin{array}{r}\text { Observations }^{+} \\
<\sim 0.05 \mathrm{pc}\end{array}$ & $\begin{array}{c}\text { Expected }{ }^{\star} \\
<0.5 \mathrm{pc} \\
\end{array}$ & \begin{tabular}{|c} 
Observations $^{+}$ \\
$<0.5 \mathrm{pc}$
\end{tabular} & Ref. \\
\hline Massive perturbers (GMC1) & $\sim 0.25$ & $66 \pm 15$ & $\sim 264$ & $212 \pm 50$ & This paper \\
\hline External binaries (Empty loss cone) & $\sim 0.76$ & $66 \pm 15$ & $\sim 87$ & $212 \pm 50$ & This paper \\
\hline External binaries (Full loss cone) & $\sim 0.12$ & $66 \pm 15$ & $\sim 539$ & $212 \pm 50$ & This paper \\
\hline Eccentric disk instability $\left(e_{\text {init }} \geq 0.7\right)$ & $\gtrsim 0.16-0.32^{1}$ & $66 \pm 15$ & $\lesssim 206-412$ & $212 \pm 50$ & \begin{tabular}{|l|l|} 
Madigan et al. 2009 \\
\end{tabular} \\
\hline Eccentric disk instability $\left(e_{i n i t}=0.6\right)$ & $\sim 0.07-0.14^{1}$ & $66 \pm 15$ & $\sim 472-943$ & $212 \pm 50$ & \begin{tabular}{|l||} 
Madigan et al. 2009 \\
\end{tabular} \\
\hline \begin{tabular}{|l|} 
Eccentric disk instability $^{2}\left(e_{\text {init }}<0.5\right)$ \\
\end{tabular} & $\lesssim 0.015-0.03^{1}$ & $66 \pm 15$ & $\gtrsim 2200-4400$ & $212 \pm 50$ & Madigan et al. 2009 \\
\hline \begin{tabular}{|l|l} 
Two disks Kozai & 3 \\
\end{tabular} & $\lesssim 0.045$ & $66 \pm 15$ & $\gtrsim 1467$ & $212 \pm 50$ & \begin{tabular}{|l|l|} 
Löckmann et al. 2009 \\
\end{tabular} \\
\hline Cluster + IMBH inspiral & $\lesssim 0.04$ & $66 \pm 15$ & $\gtrsim 1650$ & $212 \pm 50$ & Berukoff \& Hansen 2006; Fuiji et al.|2010 \\
\hline Spiral density wave $^{4}$ & $\sim 0.01$ & $66 \pm 15$ & $\sim 6600$ & $212 \pm 50$ & \begin{tabular}{|l|l|} 
Griv 2010 \\
\end{tabular} \\
\hline \multicolumn{6}{|c|}{$\begin{array}{l}\left.{ }^{+} \text {Inferred from observations (stars with } \mathrm{K} \text { magnitude } 13 \leq 14 \leq 16.5\right) \text {, Bartko et al. }(2010) \text { also H. Bartko, private communication, } 2010 \\
{ }^{\star} \text { The expected number normalized to get } \sim 53 \text { stars at }<0.05 \mathrm{pc} \\
{ }^{1} \text { The range corresponds to a range of total binary fraction in the disk, taken here to be } 0.5-1 . \\
\left.{ }^{2} \text { Several models studied, most efficient studied model is shown (in this case a disk with } e_{\text {init }}=0.5\right) \\
{ }^{3} \text { Several models studied, most efficient studied model is shown }(4 \mathrm{c} \text { in } \text { Löckmann et al. } 2009) \\
{ }^{4} \text { The total number of S-stars goes like }\left(\Delta \alpha / R_{\text {out }}\right)^{2}=(0.05 / 0.5)^{2} \sim 0.01 \text {, see Griv } 2010\end{array}$} \\
\hline
\end{tabular}

(2010), but under somewhat different conditions, who find one captured S-star $(<0.05 \mathrm{pc})$ for 21 stars stripped at $<0.4$ pc (M. Fujii, private communication, 2010). For the current population of S-stars to originate from such a scenario one requires (Eq. 1) $N_{p}=N_{s} / f_{\text {mig }} \gtrsim 1000$ B-stars to be stripped from the cluster and currently exist in the central $0.5 \mathrm{pc}$; this is much larger than the currently inferred number.

It is also difficult to envision a super-massive cluster containing $>1000$ massive B-stars within 0.06 pc of the $\mathrm{IMBH}$ for this scenario to have the necessary apriori conditions.

Even though none of the existing simulations of IMBH inspiral have been able to produce the S-cluster, including the most recent ones by Fujii et al. (2010) in which massive stars are carried down to distances no smaller than a few $0.1 \mathrm{pc}$ from the $\mathrm{MBH}$, we do note that only Berukoff \& Hansen (2006) and Fujii et al. (2010) have studied in depth the IMBH infall scenario in the context of the S-stars. Future studies may explore different conditions than those used there and may bring new insights on this subject. Similar efficiency arguments as discussed here could then be applied to test the consistency of such simulations with current observations.

\subsubsection{Binary disruption}

Another scenario where young stars are formed far from the $\mathrm{MBH}$ and then migrate close to it is the binary disruption scenario. A close pass of a binary star near a $\mathrm{MBH}$ results in an exchange interaction, in which one star is ejected at high velocity, while its companion is captured by the MBH and is left bound to it (Hills 1988). Such interaction occurs because of the tidal forces exerted by the $\mathrm{MBH}$ on the binary components. A young binary star could therefore be formed outside the central region and later be scattered onto the $\mathrm{MBH}$ on a highly radial orbit leading to its disruption. Such a scenario was suggested by Gould \& Quillen (2003) to explain the origin of the star $\mathrm{S} 2$. In order for the capture rate of such stars to explain the current observation of all the GC Bstars, rapid relaxation processes are required for the binaries to be scattered onto the MBH. Such a model, sug- gested by Perets et al. (2007), which takes into account scattering by massive perturbers outside the central 1.5 pc (such as giant molecular clouds and clumps observed in the $\mathrm{GC}$ region and other galactic nuclei; Perets et al. 2007; Perets \& Alexander 2008) could possibly account for the observed number of B-stars.

The binary disruption scenario leaves the captured stars on highly eccentric orbits $(e>0.95)$, and further dynamical evolution is required in order to explain their currently observed more relaxed eccentricity distribution. Study of their evolution, which is driven by resonant relaxation processes (Rauch \& Tremaine 1996), suggest that indeed the more relaxed, almost thermal eccentricity distribution of the S-stars (Perets et al. 2009) could be consistent with their evolution from a much higher initial eccentricity.

Note that this scenario, like the disk origin models for the S-stars, discussed above, can be constrained by observations of the parent population from which the Sstars originate. Current observations do not exclude this model (Perets et al. 2007; Perets 2009a). Future observations searching for young stars in these regions should, in principle, give better constraints. Nevertheless, other observational signatures may be more easily verified or refuted. We discuss these in the following section which focuses on this scenario.

\section{EVOLUTION OF CAPTURED STARS FROM BINARY PROGENITORS OUTSIDE THE CENTRAL PC}

In the previous section we discussed various models for the origin of the B-stars. As discussed above, most of the suggested models can be strongly constrained and even excluded by simple arguments based on current observations. In the following we focus on the binary disruption model, not yet excluded by similar arguments. In a previous study (Perets et al. 2009) we focused on the S-stars origin, and used analytic arguments and $N$-body simulations to show that a binary disruption origin followed by the (resonant) relaxation of the captured stars is consistent with their currently observed dynamical properties. Here we extend this study to explore the dynamics of the isotropic population of B-stars throughout the central pc. We first describe in more details the binary disrup- 
tion scenario. We then discuss the radial distribution of the captured B-stars according to the model and how it compares with observations. We also shortly discuss the mass function of such stars. Finally, we use $N$-body simulations and analytic arguments to find the expected eccentricity distribution of the B-stars and its dependence on the distance from the MBH. We show that the latter distribution is likely to have quite unique properties and could therefore serve as a strong constraint on the binary disruption model.

\subsection{The binary disruption scenario: details}

Young stars forming far from the MBH could be part of binaries. If such a binary is scattered into a highly eccentric orbit, it can pass the $\mathrm{MBH}$ very closely. A close pass of a such binary near the massive black hole may result in an exchange interaction, in which one star is ejected at high velocity, while its companion is captured by the MBH and is left bound to it in a tight orbit. Such interaction occurs because of the tidal forces exerted by the MBH on the binary components. Typically, a binary (with mass, $M_{b}$, and semi-major axis, $a_{b}$ ), is disrupted when it crosses the tidal radius of the $\mathrm{MBH}$ (with mass $M_{\bullet}$ ), given by

$$
r_{t}=\left(\frac{M_{\bullet}}{M_{b}}\right)^{1 / 3} a_{b}
$$

The capture probability and the semi-major axis distribution of captured stars were estimated by means of simulations, which show that most binaries approaching the $\mathrm{MBH}$ within the tidal radius $r_{t}(a)$ (Eq. 2) are actually disrupted (Hills 1991, 1992; Bromley et al. 2006). The harmonic mean semi-major axis for three-body exchanges in equal mass binaries is (Hills 1991)

$$
\left\langle a_{1}\right\rangle \simeq 0.56\left(\frac{M_{\bullet}}{M_{\text {bin }}}\right)^{2 / 3} a \simeq 0.56\left(\frac{M_{\bullet}}{M_{\text {bin }}}\right)^{1 / 3} r_{t},
$$

where $a$ is the semi-major axis of the infalling binary and $a_{1}$ that of the resulting MBH-star "binary". Most values of $a_{1}$ fall within a factor 2 of the mean. This relation maps the semi-major axis distribution of the infalling binaries to that of the captured stars: the harder (closer) the binaries, the more tightly bound the captured stars.

\subsection{Radial distribution of the B-stars}

As discussed above, the initial semi-major axis of the captured star around the $\mathrm{MBH}$ is linearly related to its binary progenitor separation (Hills 1991). The binary disruption therefore maps the the distribution of the binaries separations into the radial distribution of capture orbits. However, the binary disruption rates could also be dependent on the binary separation, implying that the cross section for disruption and hence the size of the loss cone increases with separation. Nevertheless, the latter is true only for the so-called full loss cone regime (for a detailed discussion on disruption rates and loss cone regimes see Perets et al. 2007).

In the empty loss cone regime, the disruption rate $\Gamma$ is independent of the size of the loss cone, and only depends on the relaxation time scale of the system

$$
\frac{d \Gamma}{d a} \propto \frac{d N_{b i n}(a) / d a}{t_{r}}
$$

(following Eq. 17 in Perets et al. 2007), where $N_{\text {bin }}(a)$ is the number of binaries with separation $a$ and $t_{r}$ is the relaxation time of the system. Since the separation of the captured star is linearly related to the progenitor stellar binary separation, the radial distribution of captured stars follows the binary separation distribution.

In the case of the full loss cone regime,

$$
\frac{d \Gamma}{d a} \propto \frac{d N_{b i n}(a)}{d a} q \propto \frac{d N_{b i n}(a)}{d a} a,
$$

(following Eq. 18 in Perets et al. 2007), where $q$ is the the tidal disruption radius of a given binary, which is linearly related to the binary separation (see Eq. 2 above). In this case the radial distribution of the captured stars does not directly follow the binary semi-major axis distribution.

We therefore find that the two extreme cases of empty and full loss cone regimes produce a different disruption rate dependence on the binary separation and therefore imply different radial distributions for the captured stars.

The distribution of semi-major axes of massive binaries in the Solar-neighborhood follows a log-constant distribution. In the empty loss cone regime the disruption rate is independent of the binary separations. Therefore, the radial distribution of captured stars in the GC should directly correspond to the binary separation distribution, according to Eq. 3. We therefore expect a logconstant, or a cumulative distribution of $N(<r) \propto \log r$ for the captured B-stars, under the assumption that the GC binaries have similar properties to those in the Solar neighborhood. In the full loss cone regime, the binary disruption rate is linearly dependent on the binary separation, and wider binaries have a higher chance of being disrupted compared to shorter period binaries. We therefore expect a cumulative distribution which goes like $N(<r) \propto r$.

In fig. 1 we show the cumulative distribution of semimajor axes for captured stars up to $0.5 \mathrm{pc}$, normalized to have $\sim 40$ B-stars within $0.05 \mathrm{pc}$, fairly consistent with observations (which infer $66 \pm 15$ stars; Bartko et al. (2010); also H. Bartko, private communication, 2010). Also shown in fig. 1 is the expected distribution of captured B-stars in the scenario of binary disruption induced by massive perturbers (Perets et al. 2007), which produces $\sim 40$ B-stars up to $0.05 \mathrm{pc}$. The distributions are obtained using the detailed calculations described in Perets et al. (2007), which take into account the relevant relaxation processes. The GMC1 model described there is assumed for the massive perturber scenario. A log-constant binary semi-major axis distribution is assumed for the progenitor binary population (compare with the log-normal distribution assumed in Perets et al. 2007, based on the data available at the time; Kobulnicky \& Fryer (2007), which was later revised to be a log-constant distribution). This latter scenario produces a distribution intermediate between the extreme empty loss cone regime and the extreme full loss cone regime. Interestingly, the captured stars radial distribution could therefore give a handle on the type of relaxation processes in the $\mathrm{GC}$, given the assumptions on the binaries distribution. According to GMC1 model, the expected number of B-stars in the central $0.5 \mathrm{pc}$ is $\sim 260$ stars (when noramlized to 66 at $<0.05 \mathrm{pc}$ ). The empty and full loss cone regimes, when normalized to 66 


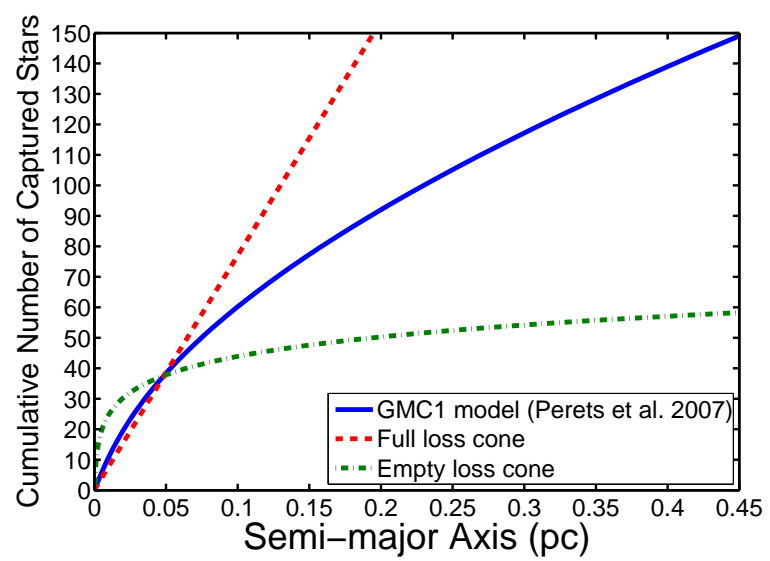

Fig. 1. - The cumulative distribution of semi-major axes for captured stars up to $0.5 \mathrm{pc}$. Several models are shown, including an empty loss cone, a massive perturbers refilled loss cone (Perets et al. 2007), and a full loss cone (see text). The empty and full loss cone models are normalized normalized to have $\sim 40$ B-stars within $0.05 \mathrm{pc}$. The massive perturbers model is not normalized, and uses the GMC1 mode (Perets et al. 2007) assumptions, but with revised binary separtions distribution (see text).

captured stars at $0.05 \mathrm{pc}$, predict, respectively, $\sim 90$ and $\sim 540$ stars captured up to $0.5 \mathrm{pc}$. In order to get a simple comparison with other models for the B-stars origin, we can define an effective "migration efficiency" for this model. In this case we replace the parent population with the population of stars captured up to 0.5 from the $\mathrm{MBH}$, i.e. strictly speaking, this is not the parent population but the stellar population which one would like to probe when comparing the models studied here. We find in this case $f_{m i g} \sim 0.25$ and $\sim 0.12$ for the empty and full loss cone regimes, respectively (see also table 10).

\subsection{Eccentricity distribution of the B-stars}

The periapse of the captured star in the binary disruption scenario is at $r_{t}$, and therefore its eccentricity is very high (Hills 1991; Miller et al. 2005),

$$
e=1-r_{t} / a_{1} \simeq 1-1.8\left(M_{\mathrm{bin}} / M_{\bullet}\right)^{1 / 3} \gtrsim 0.97
$$

for values typical of binaries and the $\mathrm{MBH}$ in the $\mathrm{GC}$ $\left(M_{\text {bin }}=20 M_{\odot} ; M_{\bullet}=4 \times 10^{6} M_{\odot}\right)$.

For comparison, the initial eccentricity distribution of stars formed in a stellar disk is likely to be biased to much more circular orbits. For example, the currently observed eccentricity distribution of the young $\mathrm{O}$ and WR stars observed in the clockwise disk peaks at $e \sim 0.35$.

It is interesting to note, however, that the resonance relaxation timescales in the $\mathrm{GC}$ increase with distance from the MBH (Hopman \& Alexander 2006a). Therefore, stars captured/formed further away from the $\mathrm{MBH}$ are likely to have a less relaxed eccentricity distribution, i.e. over time we may expect a correlated eccentricitydistance distribution to be produced, with stars more distant from the $\mathrm{MBH}$ having an eccentricity distribution which much more closely resembles the original one. Far from the $\mathrm{MBH}$, where the resonant relaxation timescales are much larger than the lifetimes of the B-stars (see e.g. Hopman \& Alexander 2006a), stars should keep their original eccentricity distribution, i.e. highly eccentric orbits for captured stars, and likely low eccentricity orbits for stars formed in a stellar disk. Stars captured a few $0.1 \mathrm{pc}$ away from the $\mathrm{MBH}$, for example, are likely to still conserve their initial eccentricities. Closer to the MBH captured stars could have a relaxed (thermal eccentricity) distribution even after short times, although the captured stars population relaxes to a thermal eccentricity distribution much faster than a stellar population with initially low typical eccentricity (Perets et al. 2009)).

\subsubsection{Results of $N$-body simulations}

In order to study the eccentricity distribution of the captured stars and its radial dependence more quantitatively we performed detailed $N$-body simulations. We used the $\phi G R A P E$ direct summation software running on gravitySimulator, the 32-node cluster at the Rochester Institute of Technology that incorporates GRAPE accelerator boards in each of the nodes (Harfst et al. 2007). We assume that the main contribution to the dynamical relaxation of stars in the GC comes from stellar black holes (SBHs). We consider a distribution of SBHs following the results of Fokker-Plank calculations of the distribution of $10 M_{\odot}$ SBHs around a $\mathrm{MBH}$ found by (Hopman \& Alexander 2006b). We use $16000 \mathrm{SBHs}$ of $10 M_{\odot}$ with an isotropic radial, $r^{-2}$, distribution between $0.04 \mathrm{pc}$ and $0.8 \mathrm{pc}$ around a $\mathrm{MBH}$ of $3.6 \times 10^{6} M_{\odot}$ (the simulation of stars closer to the MBH requires much longer simulation time; we discussed the evolution of such stars in Perets et al. 2009). In order not to be affected by boundary effects we focus on stars in the range $0.08--0.5 \mathrm{pc}$ from the $\mathrm{MBH}$. In all of our simulations we force the $\mathrm{MBH}$ to be at rest at the origin of the system since the Brownian motion is expected to be quite low (Merritt 2005). Note that the full cusp of low mass stars was not included in our simulations (this is beyond our current computatational capabilites). Such stars could somewhat accelarate the resonant relaxation processes as more stars contribute to the process. However, the larger mass contributing to the cusp potential would also produce precession of the stellar orbits, thereby producing enhanced quenching of resonant resonant relaxation. The neglection of the full cusp potential therefore suggests that our N-body results likely give an upper limit to the relaxation rate due to resonant relaxation. The signature of the initial capture conditions might therefore even better preserved than suggested by the current simulations. As in our previous simulations (Perets et al. 2009) general relativisitic precession due to the $\mathrm{MBH}$ is not included. The latter component, however, is not important for stars at the distances from the $\mathrm{MBH}$ discussed here.

The "captured stars" followed by our simulations are those stars with initial eccentricities of $0.95 \leq e \leq 0.99$.

In fig. 2 we show the temporal evolution of the median eccentricity of the captured B-stars in the central $0.5 \mathrm{pc}$, at various distances from the $\mathrm{MBH}$. The MS lifetimes of $7-15 M_{\odot}$ stars are $\sim 10-50$ Myrs. in the future.

In figs. 3 - 3 - we show the cumulative eccentricity distribution in three different distance regimes and at 20, 40 and 60 Myrs of evolution. Also shown for comparison is a thermal eccentricity distribution $(f(e)=2 e$; cumulative $\left.c f(e)=e^{2}\right)$.

In fig. 4 we show the median eccentricity of the captured B-stars vs. their separation from the $\mathrm{MBH}$ at 20, 


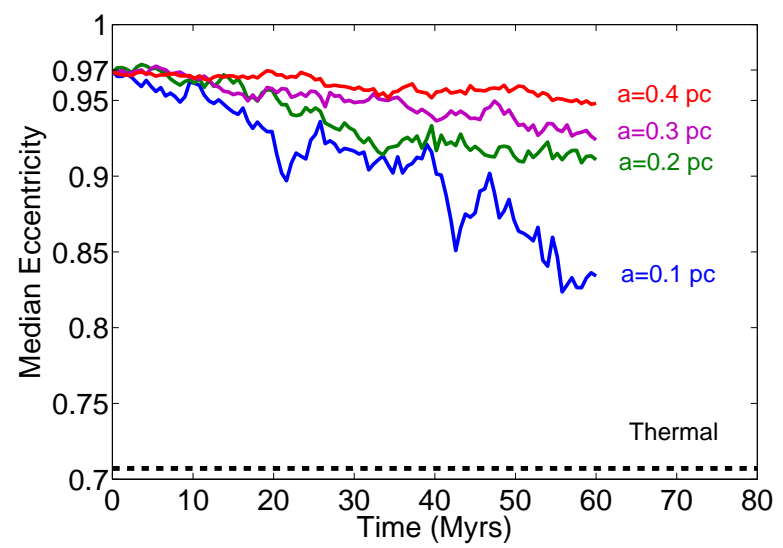

FIG. 2.- The temporal evolution of the median eccentricity of the captured B-stars in the central $0.5 \mathrm{pc}$, at various distances from the $\mathrm{MBH}$.

40 and 60 Myrs of evolution. As can be seen in this figure, the binary capture scenario provides a qualitatively unique signature, in which the typical eccentricity of the stars rises with distance. The exact relaxation rate in the GC is dependent on the number density of stars and SBHs in this environment, which is still poorly constrained. For our assumptions on the number density of SBHs, captured stars a few 0.1 pc away from the $\mathrm{MBH}$ have very high $(>0.9)$ eccentricities, whereas stars are more relaxed at closer to the $\mathrm{MBH}$, as also found in previous studies on the captured population even closer to the MBH (Perets et al. 2009). Faster relaxation rates (e.g produced by a higher density of SBHs and/or the additional contribution to relaxation process by low mass stars, not included here) would still produce the qualitative signature of a typical eccentricity rising with distance, but may quantitatively show lower eccentricities, and a relaxed population up to larger distances from the $\mathrm{MBH}$. Conversely, a stellar population with initially low $(<0.5)$ eccentricities, as might be expected from a stellar disk population, may show the opposite picture, with progressively lower eccentricities at larger distances from the $\mathrm{MBH}$. For this reason the distribution of eccentricities vs. distance could be a key observational property for discriminating models for the origin of the young Bstars. We do note, however, that very close to the $\mathrm{MBH}$ (the innere regions of the S-cluster art a few 0.1 pcs from the $\mathrm{MBH}$ ), where general relativistic precession due to the $\mathrm{MBH}$ becomes important. At these regions resonant relaxation might be quenched, and capture stars could again show higher eccentricities.

\subsection{Mass function of the captured stars}

The mass function (MF) of captured stars is likely to be regular at the high mass regime $\left(\gtrsim 3 M_{\odot}\right)$, i.e. reflecting the mass function of stars far from the MBH, where regular star-formation can take place, and where the progenitor binaries form. The currently observed MF, however, may slightly differ from the regular MF due to several reasons.

(a) Binary fractions and the distribution of binary orbital properties are mass dependent. Massive (OB) stars have higher binary fractions than lower mass stars. Massive binaries are also typically much more compact (a log constant distribution of periods vs. a log-normal distribution for low mass stars; e.g. Duquennoy \& Mayor 1991; Kobulnicky \& Frver 2007). The binary disruption process requires binary progenitors for the captured stars. It also maps the binary period into the capture separation from the $\mathrm{MBH}$ as discussed above. For both these reasons, a larger fraction of massive stars is likely to be captured in this scenario, compared with the fraction of low mass stars binary disruption (relative fractions; in terms of absolute frequency of low mass binary stars, they are still more frequent than high mass binary stars). In addition, massive stars are typically captured much closer to the $\mathrm{MBH}$ than low mass stars. Therefore, the population of captured stars in the GC, especially in the central pc, tends to be biased towards massive $\mathrm{OB}$ stars.

(b) Longer living stars (hence lower mass stars) captured at earlier times may have a higher probability of being disrupted by the MBH during their dynamical evolution (Perets et al. 2009). On even longer timescales (twobody relaxation timescales), they can migrate from their original capture orbit. For this reason, the observed population of captured stars is biased towards stars which were recently captured, i.e. selected against long living stars captured at earlier times.

(c) A small contribution from Kozai induced merger of captured binary stars, induced by Kozai resonances near the $\mathrm{MBH}$, may contribute a small fraction of more massive stars (Antonini et al. 2009; Perets 2009b; Perets \& Fabrycky 2009).

Currently, the MF of the probed B-stars population is consistent with a continuous star formation (with regular IMF) over the last $\sim 50$ Myrs (Bartko et al. 2010). The range of probed masses is currently quite limited, and low mass stars are not included in the analysis (and therefore point (a) is not directly verified, given our limited knowledge on the binary distribution of OB stars of different masses. For this limited range, the observed MF is qualitatively consistent with the expected one. Future observations probing fainter stars will provide much better data and therefore stronger constraints on the mass function.

\section{DISCUSSION}

Any model suggesting a common origin for all/most of the B-stars in the $\mathrm{GC}$ is required to produce both the stars in the inner region $(<0.05 \mathrm{pc})$ and those in the outer region $(<0.5 \mathrm{pc}$ or more). Most of our discussion on the constraints on different models for the origin of these stars can therefore be summarized by two simple qualitative properties: (i) the ratio between the number of stars in the inner region and the outer region of the $\mathrm{GC}$, (ii) the radial eccentricity distribution trend in these regions.

Table 1 summarizes the predictions of the various theoretical models for the number of B-stars in the inner/outer regions, compared to current observations. As can be seen, most models grossly over-predict the number of B-stars in the outer region, up to $0.5 \mathrm{pc}$, given the observed number of B-stars in the central 0.05 pc. Such constraints could easily be studied and extended/generalized for any new model suggested for the B-stars origin.

Currently, we find that the most consistent explanation 

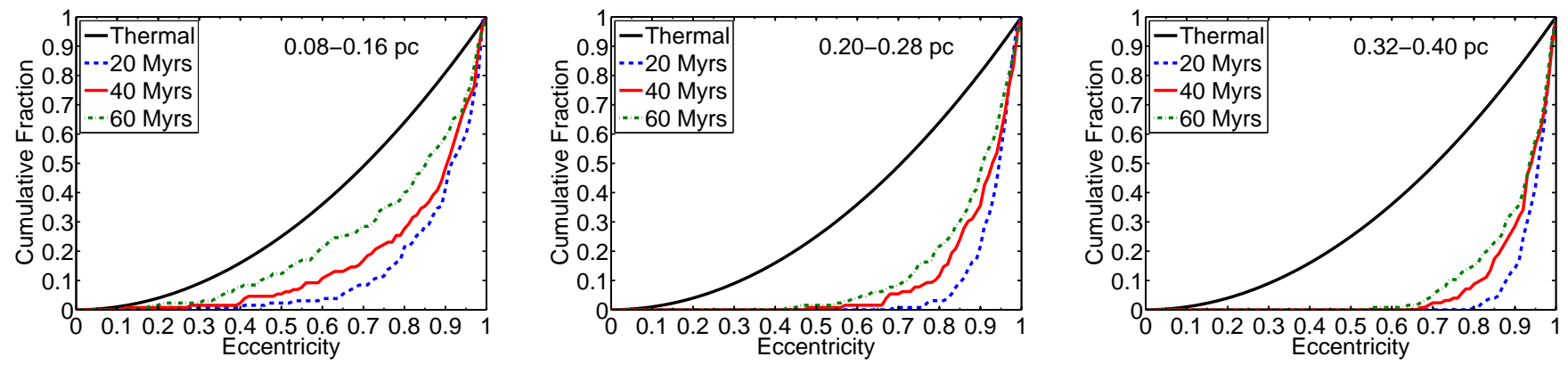

FIG. 3.- The cumulative eccentricity distribution in three different distance regimes, after 20, 40 and 60 Myrs of evolution. Also shown for comparison is a thermal eccentricity distribution $\left(f(e)=2 e\right.$; cumulative $\left.c f(e)=e^{2}\right)$. Captured stars have initial eccentricities between 0.95 and 0.99 .

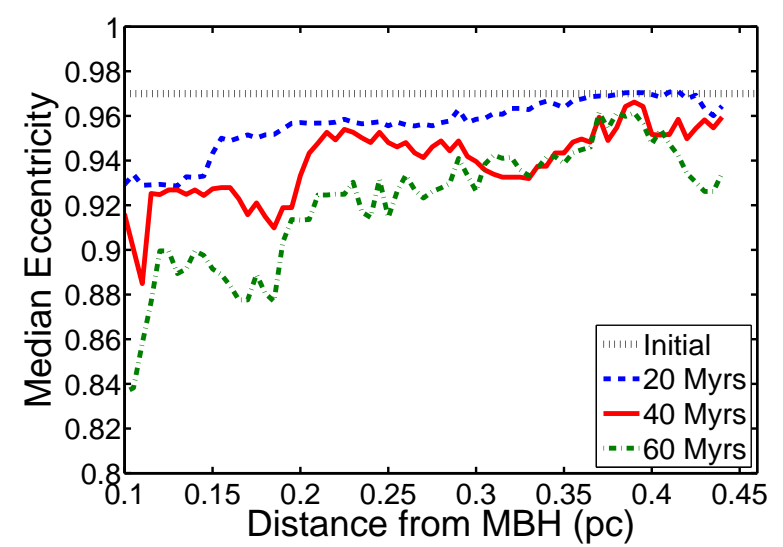

Fig. 4.- The median eccentricity of the captured B-stars vs. their separation from the MBH after 20, 40 and 60 Myrs of evolution. For comparison the median eccentricity of a relaxed thermal population is $\sim 0.7(\sqrt{(1 / 2)}$. Captured stars have initial median eccentricity of $\sim 0.97$ (top dotted line).

for the complete population of B-stars in the GC is an external formation followed by binary disruption (likely driven by massive perturbers). The eccentricity distribution of the captured stars in this scenario should be dependent on the distance from the MBH. Higher eccentricities should be observed for stars captured at larger distances.

The formation of binary B-stars in a very eccentric stellar disk with $e_{\text {init }}>0.7$ could also be consistent with the ratio of inner to outer B-stars, for a high enough binary fraction in the stellar disk. The radial distribution of the stars in this case is likely to differ from that of the massive perturbers scenario, as the population of B-stars would include both the progenitor population formed in the stellar disk and the captured stars. These two populations are likely to have different radial distributions, and their combined distribution is likely to show a bimodality, with one peak at the inner region close to the $\mathrm{MBH}$, dominated by the captured B-stars, and an additional peak corresponding to the peak in the stellar disk. From the simulations by Madigan et al. (2009), it seems that the binary disruption is only weakly dependent on the binary separation. This is reminiscent of the empty loss cone regime discussed above. The radial distribution of the captured stars should therefore correspond to a direct mapping of the binaries separations distribution in this case. The eccentricity distribution of the B-stars should also be a combination of two populations. A more detailed study of this scenario, however, is beyond the scope of this paper and will be studied elsewhere (see Madigan et al. 2009 for a discussion of the basic scenario).

\section{SUMMARY}

In this study we explored the dynamical constraints on the origin of the young B-stars in the Galactic center. We showed that most of the currently studied models could not consistently explain both the population of the S-stars (the B-stars at $<0.05 \mathrm{pc}$ from the $\mathrm{MBH}$ ), and the extended population of young B-stars up to $0.5 \mathrm{pc}$. In these models the number of B-stars in the inner and outer regions are incompatible.

We used analytical arguments and N-body simulations to study the capture and the dynamics of the B-stars in the external formation+binary disruption scenario, which is one of the models consistent with the observed B-stars numbers at different regions of the GC. Using our analysis we provide detailed predictions for the distribution of the B-stars dynamical properties, which could serve as observational signatures to further support, constrain or refute this model.

We would like to thank Tal Alexander for helpful discussions as well as Hendrik Bartko and Michiko Fujii for providing us with data from their observations and simulations, respectively. We would also like to thank David Merritt for the use of the GRAPE cluster at the Rochester Institute of Technology. HBP would like to thank the Israeli Science Foundation, the Bi-national Fulbright program and the Israeli industrial and commercial club for their support through the BIKURA (FIRST) and Ilan Ramon-Fulbright fellowships. AG is supported by grant NNX07AH15G from NASA.

\section{REFERENCES}

Alexander, R. D., Begelman, M. C., \& Armitage, P. J. 2007, ApJ, 654,907

Alexander, T. \& Livio, M. 2004, ApJl, 606, L21
Antonini, F. et al. 2009, arXiv:0909.1959

Bartko, H. et al. 2009, ApJ, 697, 1741

—. 2010, ApJ, 708, 834 
Berukoff, S. J. \& Hansen, B. M. S. 2006, ApJ, 650, 901

Bonnell, I. A. \& Rice, W. K. M. 2008, Science, 321, 1060

Bromley, B. C. et al. 2006, ApJ, 653, 1194

Buchholz, R. M. et al. 2009, A\&A, 499, 483

Cuadra, J., Armitage, P. J., \& Alexander, R. D. 2008, MNRAS, 388, L64

Do, T. et al. 2009, ApJ, 703, 1323

Duquennoy, A. \& Mayor, M. 1991, A\&A, 248, 485

Eisenhauer, F. et al. 2005, ApJ, 628, 246

Fujii, M. et al. 2008, ApJ, 686, 1082

—. 2009, ApJ, 695, 1421

—. 2010, arXiv:1003.4125

Gerhard, O. 2001, ApJl, 546, L39

Ghez, A. M. et al. 1998, ApJ, 509, 678

Gillessen, S., Eisenhauer, F., Trippe, S., Alexander, T., Genzel, R., Martins, F., \& Ott, T. 2009, ApJ, 692, 1075

Glebbeek, E. et al. 2009, A\&A, 497, 255

Gould, A. \& Quillen, A. C. 2003, ApJ, 592, 935

Griv, E. 2010, ApJ, 709, 597

Gualandris, A. \& Merritt, D. 2009, ApJ, 705, 361

Gürkan, M. A., Freitag, M., \& Rasio, F. A. 2004, ApJ, 604, 632

Gürkan, M. A. \& Rasio, F. A. 2005, ApJ, 628, 236

Hansen, B. M. S. \& Milosavljević, M. 2003, ApJl, 593, L77

Harfst, S. et al. 2007, New Astronomy, 12, 357

Hills, J. G. 1988, Nature, 331, 687

-. 1991, AJ, 102, 704

-. 1992, AJ, 103, 1955

Hobbs, A. \& Nayakshin, S. 2009, MNRAS, 394, 191

Hopman, C. \& Alexander, T. 2006a, ApJ, 645, 1152

-. 2006b, ApJl, 645, L133

Kim, S. S., Figer, D. F., \& Morris, M. 2004, ApJ, 617, L123

Kim, S. S. \& Morris, M. 2003, ApJ, 597, 312

Kobulnicky, H. A. \& Fryer, C. L. 2007, ApJ, 670, 747

Levin, Y. 2007, MNRAS, 374, 515
Levin, Y. \& Beloborodov, A. M. 2003, ApJl, 590, L33

Levin, Y., Wu, A., \& Thommes, E. 2005, ApJ, 635, 341

Löckmann, U. \& Baumgardt, H. 2008, MNRAS, 384, 323

Löckmann, U., Baumgardt, H., \& Kroupa, P. 2009, MNRAS, 398, 429

Lu, J. R. et al. 2009, ApJ, 690, 1463

Madigan, A.-M., Levin, Y., \& Hopman, C. 2009, ApJl, 697, L44

Merritt, D. 2005, ApJ, 628, 673

—. 2009, ArXiv:0909.1318

Merritt, D., Gualandris, A., \& Mikkola, S. 2009, ApJl, 693, L35

Miller, M. C. et al. 2005, ApJl, 631, L117

Morris, M. 1993, ApJ, 408, 496

Nayakshin, S. \& Cuadra, J. 2005, A\&A, 437, 437

Paumard, T. et al. 2006, ApJ, 643, 1011

Perets, H. B. 2009a, ApJ, 690, 795

-. 2009b, ApJ, 698, 1330

Perets, H. B. \& Alexander, T. 2008, ApJ, 677, 146

Perets, H. B. \& Fabrycky, D. C. 2009, ApJ, 697, 1048

Perets, H. B., Hopman, C., \& Alexander, T. 2007, ApJ, 656, 709

Perets, H. B., Kupi, G., \& Alexander, T. 2008a, in IAU Symposium, Vol. 246, IAU Symposium, ed. E. Vesperini, M. Giersz, \& A. Sills, 275-276

Perets, H. B. et al. 2008b, Memorie della Societa Astronomica Italiana, 79, 1100

-. 2009, ApJ, 702, 884

Portegies Zwart, S. F., McMillan, S. L. W., \& Gerhard, O. 2003, ApJ, 593, 352

Rauch, K. P. \& Tremaine, S. 1996, New Astronomy, 1, 149

Vanbeveren, D. et al. 2009, APSS, 324, 271

Wardle, M. \& Yusef-Zadeh, F. 2008, ApJL, 683, L37

Yu, Q., Lu, Y., \& Lin, D. N. C. 2007, ApJ, 666, 919

Yungelson, L. R. et al. 2008, A\&A, 477, 223 